\title{
Habilidades comunicativas de los residentes con las familias de bebés con síndrome de Down
}

\author{
Communication skills of residents to families with \\ Down syndrome babies
}

\author{
Prof. Asoc. Dra. Derya G. Dogan ${ }^{a}$ Dra. Yesim Kutluturk ${ }^{b}$ Dra. Meltem Kivilcim ${ }^{b}$ y \\ Sinem K. Canaloglu, MSc ${ }^{a}$
}

\section{RESUMEN}

Antecedentes. En general, los pediatras son los primeros profesionales de la atención médica encargados de dar el diagnóstico inicial de síndrome de Down (SD) a las familias. Sin embargo, cuando un bebénace conSD, la mayoría de los padres no se sienten satisfechos con el contenido de la información brindada ni con la forma en que la recibieron. Los residentes de pediatría deberían participar en intervenciones educativas para ayudar a los padres a superar estos problemas y brindarles información precisa. El objetivo de este estudio es evaluar los niveles de comodidad de los residentes de pediatría y la necesidad de capacitación para comunicarse con los padres de niños que nacen con SD.

Métodos. El Inventario dela situación diagnóstica (ISD) es una escala de calificación de diez preguntas que evalúa el nivel de incomodidad. Se envió una encuesta a todos los residentes de pediatría del país a través de la telefonía celular, sitios web y todos los medios sociales disponibles. También se registraron los factores sociodemográficos, incluida la información sobre los requisitos de capacitación.

Resultados. Entre los 326 participantes, el nivel de incomodidad medio total fue del 30,22 sobre 50, el puntaje más alto del ISD. El nivel de incomodidad fue significativamente más alto entre las mujeres $(p=0,033)$. Se observó que ser mujer $(p=0,014)$, estar en uno de los primeros años de residencia $(p=0,028)$, haber examinado a una menor cantidad de pacientes con SD $(p=0,025)$ y sentir un nivel mayor de incomodidad $(p=0,001)$ son factores relacionados con una mayor necesidad de capacitación. El $84 \%$ de los residentes indicaron que necesitan capacitación adicional.

Conclusiones. Este estudio mostró que los residentes de pediatría tuvieron un nivel de incomodidad alto cuando comunicaban el diagnóstico de SD a los padres del recién nacido. Las residentes tuvieron un nivel de incomodidad significativamente mayor que los residentes. Palabras clave: residente de pediatría, sindrome de Down, diagnóstico, habilidades comunicativas, capacitación.

http:/ /dx.doi.org/10.5546/aap.2016.521

Texto completo en inglés:

http://dx.doi.org/10.5546/aap.2016.eng.521

\section{INTRODUCCIÓN}

El síndrome de Down (SD) es el trastorno genético más frecuente: 1 de cada 800 nacidos vivos tienen $\mathrm{SD},{ }^{1}$ y más del $85 \%$ de las madres toman conocimiento del diagnóstico después del nacimiento. ${ }^{2}$ En general, los pediatras son los primeros profesionales de la atención médica encargados de dar el diagnóstico de SD a las familias en los países donde el asesoramiento genético no está extendido, y están en posición de ayudar a los padres a sobreponerse al primer impacto provocado por el diagnóstico de SD en sus bebés. Según los informes, incluso veinte años después, las familias suelen recordar las primeras palabras dichas por los médicos durante la conversación sobre el diagnóstico inicial. ${ }^{3}$ Desafortunadamente, las familias no se sintieron satisfechas con la información que recibieron sobre tener un bebé con SD, ni con el contenido y la forma en que se les brindó. ${ }^{4,5}$

La diversidad entre las culturas, los antecedentes educativos y los diferentes dialectos son sumamente importantes a la hora de comunicar información genética. ${ }^{6}$ Se desconoce cómo se sienten los residentes de pediatría a la hora de dar la noticia del diagnóstico de SD o cómo se comportan frente a las familias.

Hasta donde se sabe, para evaluar las habilidades comunicativas, se ha desarrollado solamente un instrumento: el Inventario de la situación diagnóstica (ISD), que 
examina la actitud de los residentes al dar el diagnóstico de SD en un recién nacido a la familia. El ISD fue desarrollado por Ferguson y col. (EE. UU.) y ha sido utilizado para mejorar, entre los residentes de obstetricia y pediatría, el nivel de entendimiento respecto de los problemas, los niveles de comodidad y las necesidades de los pacientes en relación con la prueba de detección y el diagnóstico de SD. ${ }^{7}$

La meta final de este estudio fue generar una mejor percepción sobre cómo brindar información de manera sensible y adecuada a las familias de los bebés con diagnóstico de SD entre los residentes de pediatría de los países del Este.

El objetivo de este estudio era evaluar los niveles de comodidad y los requisitos de capacitación de los residentes de pediatría para comunicarse con los padres de los bebés con SD.

\section{MÉTODOS}

Esta fue una encuesta transversal autoadministrada y enviada por correo. Se diseñó el ISD en el sitio web surveymonkey.com y se distribuyó entre los residentes de pediatría junto con una carta de presentación a través de la telefonía celular, sitios web y medios sociales en dos instancias entre enero y febrero de 2015. También se envió un recordatorio a los residentes que no hubieran respondido al cuestionario en el término de dos semanas. En la carta de presentación se explicaba el objetivo del estudio y se destacaba el hecho de que la participación era absolutamente voluntaria. El criterio de inclusión era ser residente de pediatría en Turquía al momento de la encuesta. Se excluyó a los residentes graduados antes del 2000 y después del 2014. El tamaño de la muestra se calculó mediante un análisis de potencia, y se estableció que debía ser de, al menos, 128 participantes.

A partir de nueve preguntas hechas antes de comenzar a aplicar el ISD, se obtuvo la siguiente información: datos demográficos, sexo, año de residencia, estado civil y cantidad de niños con $\mathrm{SD}$ evaluados durante la residencia. Además se hizo una pregunta para establecer si el residente deseaba o no recibir capacitación en este campo.

El nivel de comodidad de los residentes de pediatría al dar un diagnóstico de síndrome de Down se evaluó con el "Inventario de la situación diagnóstica", con el permiso del autor original. En este caso, se utilizó el inventario actualizado por Lunney y col. en 2012. ${ }^{8}$

El ISD refleja diversos grados de ambigüedad en muchos desafíos relacionados con el diagnóstico de SD. Cinco de los componentes del ISD miden el nivel de incomodidad al recibir preguntas ambiguas desde el punto de vista médico para las cuales no existen respuestas médicas claras, y los otros cinco miden el nivel de incomodidad ante preguntas inequívocas que pueden responderse con información más bien fáctica. El inventario está preparado para residentes de obstetricia y pediatría; por ello, la décima pregunta ("Una paciente a la que le dijeron que la prueba de detección indica que tiene un riesgo mayor de tener un niño con síndrome de Down decide no realizarse la prueba diagnóstica definitiva"), que se refiere al diagnóstico prenatal, se cambió, con el permiso del autor, por una pregunta sobre el cuidado personal ("Un padre pregunta si su hijo podrá asearse por su cuenta, al igual que otros niños sanos"). Las respuestas de cada elemento se puntuaron usando la escala de Likert, donde " 1 " significaba "Para nada incómodo" y "5", "Sumamente incómodo". El

TABLA 1: Características sociodemográficas de los residentes de pediatría ( $n$ : 326)

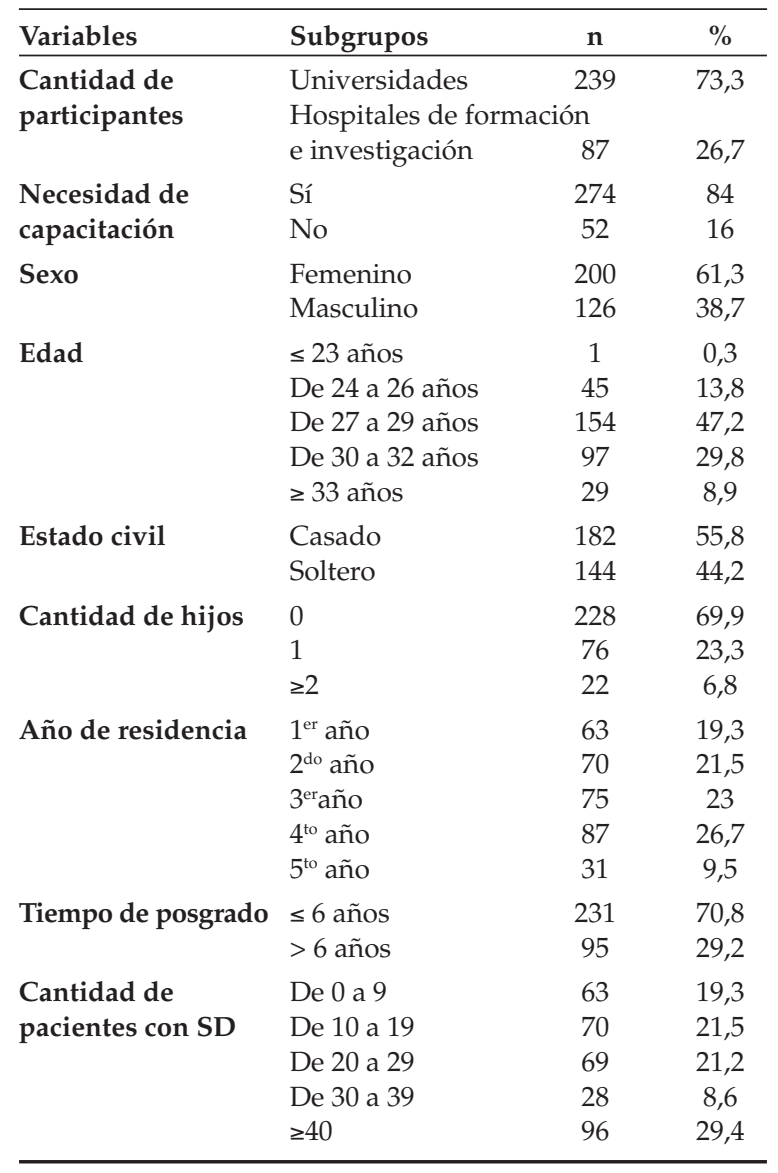


puntaje de incomodidad total máximo posible era de 50, con un intervalo potencial de 10 a 50 . Los puntajes por encima de los puntajes medios se consideraron como un mayor nivel de incomodidad.

El protocolo del estudio fue aprobado por el Comité de Ética de la İnönü Üniversitesi.

El análisis estadístico se realizó con el programa SPSS 17.0. Los resultados se expresaron como datos estadísticos descriptivos. Los datos se analizaron con la prueba de $\chi^{2}$ de Pearson para los datos no correlacionados y la prueba $t$ para los datos correlacionados. Como método de análisis, se usaron la media y la desviación estándar. Los valores de $p<0,05$ se consideraron significativos.

\section{RESULTADOS}

Se enviaron correos electrónicos a 1000 residentes; de ellos, respondieron 341 . En el 2015, había 2441 residentes de pediatría (1368 en hospitales universitarios y 1073 en hospitales de formación e investigación). La tasa de respuesta fue del $34,1 \%$. Se excluyó a 15 participantes debido a datos imprecisos o faltantes, por lo que el estudio incluyó a 326 residentes que respondieron la encuesta. Eran
$239(73,3 \%)$ residentes de pediatría de hospitales universitarios y $87(26,7 \%)$ de hospitales de formación e investigación. Del total de residentes, el 61,3\% eran mujeres, y el 19,3\% estaban en los primeros años de la residencia. En la Tabla 1, se presentan las características sociodemográficas de los participantes.

El nivel de incomodidad medio total fue del 30,22 $\pm 7,21$ según el ISD, sobre 50, que era el puntaje máximo. El puntaje medio de las preguntas ambiguas fue del $17,77 \pm 3,73$ y de las preguntas inequívocas, del 12,45 $\pm 4,19$.

El promedio del puntaje medio de cada componente fue 2,60. La pregunta sobre el consejo relacionado con el mayor riesgo de problemas cardíacos fue la que obtuvo el puntaje medio más bajo $(2,06 \pm 0,96)$, mientras que la pregunta sobre la madre que escucha a un colega decir: "Los niños con síndrome de Down nunca logran nada" fue la que tuvo el puntaje medio más alto $(4,16 \pm 0,99)$. En la Tabla 2 , se muestran los puntajes medios de todos los componentes.

El nivel medio de incomodidad $(p=0,033)$ y el nivel total de ambigüedad $(p=0,008)$ fueron significativamente más altos entre las residentes mujeres que entre los varones, según el método

TABla 2: Componentes del Inventario de la situación diagnóstica y puntajes del nivel de incomodidad

\begin{tabular}{|c|c|c|}
\hline $\begin{array}{l}\text { Inventario de la situación diagnóstica } \\
\text { Preguntas }\end{array}$ & $\begin{array}{l}\text { Nivel medio de } \\
\text { incomodidad previo }\end{array}$ & DE \\
\hline $\begin{array}{l}\text { 1) Los padres se desmoronan al escuchar el diagnóstico (en el consultorio del médico) y } \\
\text { se preguntan: ¿Por qué a nosotros?". }\end{array}$ & 3,21 & 0,98 \\
\hline $\begin{array}{l}\text { 2) La madre pregunta si criar a un niño con síndrome de Down será muy diferente } \\
\text { de criar a otros niños. }\end{array}$ & 2,91 & 0,99 \\
\hline $\begin{array}{l}\text { 3) El padre parece consternado y dice que la familia no cuenta con los recursos para } \\
\text { encargarse de un niño con síndrome de Down. }\end{array}$ & 3,80 & 0,99 \\
\hline $\begin{array}{l}\text { 4) Después de recibir el diagnóstico de síndrome de Down del bebé recién nacido, } \\
\text { ambos padres parecen estar muy deprimidos y no hablan ni hacen preguntas. }\end{array}$ & 3,69 & 0,94 \\
\hline $\begin{array}{l}\text { 5) La madre llega al consultorio llorando porque escuchó a un colega suyo decir: } \\
\text { "Los niños con síndrome de Down nunca logran nada". }\end{array}$ & 4,16 & 0,99 \\
\hline Total de las preguntas ambiguas (1-5) & 17,77 & 3,73 \\
\hline $\begin{array}{l}\text { 6) Uno de los padres pregunta si el niño podrá asistir a una escuela normal y } \\
\text { aprender junto a los otros niños. }\end{array}$ & 2,79 & 1,06 \\
\hline $\begin{array}{l}\text { 7) Los padres preguntan si existe un riesgo mayor de tener un segundo hijo } \\
\text { con síndrome de Down en caso de concebir nuevamente. }\end{array}$ & 2,17 & 1,06 \\
\hline $\begin{array}{l}\text { 8) Los padres están preocupados por el mayor riesgo de problemas cardíacos y } \\
\text { quieren un consejo sobre qué hacer. }\end{array}$ & 2,06 & 0,96 \\
\hline $\begin{array}{l}\text { 9) La madre se rehúsa a creer que su bebé tiene síndrome de Down porque tiene solo } 25 \text { años y } \\
\text { "las madres jóvenes no tienen hijos con síndrome de Down". }\end{array}$ & 3,01 & 1,25 \\
\hline $\begin{array}{l}\text { 10)Uno de los padres pregunta si el niño podrá asearse por su cuenta, } \\
\text { al igual que otros niños sanos. }\end{array}$ & 2,43 & 1,09 \\
\hline Total de las preguntas inequívocas (6-10) & 12,45 & 4,19 \\
\hline
\end{tabular}


estadístico de la prueba de $\chi^{2}$ de Pearson. Entre las doctoras, $176(53,99 \%)$ indicaron que requerían más capacitación, en comparación con los doctores $(98[30 \%])(p=0,014)$.

La prueba $t$ independiente indicó que ser mujer $(p=0,014)$, estar en uno de los primeros años de residencia $(p=0,028)$, haber examinado a una menor cantidad de pacientes con SD $(p=0,025)$ y sentir un nivel mayor de incomodidad $(p=0,001)$ son factores relacionados con un mayor requisito de capacitación. El año de residencia medio era $2,79 \pm 1,28$ en el grupo que requería capacitación y 3,21 $\pm 1,17$ en el grupo que no requería capacitación $(p=0,03)$. Con el mismo método estadístico, se estableció que la cantidad media de pacientes con SD examinados era del $29,9 \pm 1,50$ en el grupo que requería capacitación y $35 \pm 1,43$ en el grupo que no requería capacitación $(p=0,03)$.

La mayoría de los participantes de los hospitales universitarios $(84,1 \%)$ y de 10 s hospitales de formación e investigación $(83,9 \%)$ indicaron requerir capacitación adicional sobre cómo informar a las familias que tienen bebés con síndrome de Down.

\section{DISCUSIÓN}

Con este estudio se demostró que los residentes de pediatría de un país oriental no se sienten cómodos al dar la información sobre el diagnóstico de SD a las familias de los recién nacidos. Ser una residente de sexo femenino, estar en los primeros tres años de la residencia y haber examinado a menos de 30 pacientes con SD estuvieron asociados con un mayor nivel de incomodidad y más requisitos de capacitación.

El ISD se desarrolló en Kentucky University para mejorar las incompetencias de los residentes de obstetricia y ginecología y de pediatría durante el asesoramiento a los padres de los bebés con SD. Se evaluaron los conocimientos y niveles de comodidad de los residentes con una prueba realizada antes y después de una intervención educativa con CD-ROM. ${ }^{7}$ Después de seis años, Lunney y col., modificaron el ISD, que ha sido aceptado como investigación con un tutorial en línea. En ese estudio, Lunney observó que el nivel medio de incomodidad de los residentes era del 23,05 sobre un nivel de incomodidad máximo de $35 .{ }^{8} \mathrm{El}$ nivel medio de incomodidad de los residentes turcos fue del 30,22 sobre un nivel máximo de 50. El mayor nivel de insatisfacción de los residentes turcos se debe probablemente a que no reciben capacitación clínica estructurada sobre cómo dar malas noticias a las familias.

En el estudio de Lunney, el 97\% de los residentes declararon que la capacitación era necesaria; por el contrario, el $84 \%$ de los residentes turcos indicaron que deseaban recibir capacitación. Dado que en el estudio de Lunney los conocimientos y niveles de incomodidad de los residentes se evaluaron con una prueba realizada antes y después de un programa educativo, es posible que los residentes hayan tenido una mayor predisposición a recibir capacitación. ${ }^{8}$

En este estudio, el nivel de incomodidad de las residentes mujeres era mayor que el de los hombres, y se observó que estaban más dispuestas a recibir capacitación. La diferencia entre sexos no se menciona en el estudio de Lunney. ${ }^{8}$ Diversos estudios demostraron que las habilidades comunicativas difieren entre doctoras y doctores. Se sabe que las doctoras tienen un mejor manejo de los pacientes debido a su buena comunicación. ${ }^{9}$ En un metanálisis, Roter y col. descubrieron que las doctoras se involucran en la conversación más positivamente (por ejemplo, brindando apoyo), hacen preguntas más bien de tipo psicosocial, hablan más sobre las emociones y demuestran un comportamiento que apunta más a establecer una asociación. ${ }^{10}$

Al mantener una comunicación adecuada y prestar servicios sanitarios y comunitarios, es posible que los pediatras tengan un mayor apego con los pacientes y sus familias. Estas desean obtener información actualizada, precisa y ecuánime. ${ }^{5,11,12} \mathrm{Si}$ las familias reciben información de profesionales médicos confiables, tal vez se sientan más optimistas sobre el hecho de tener un bebé con SD. Los sentimientos optimistas fomentan una relación sana entre padres e hijos y optimizan las emociones, el crecimiento y el desarrollo. ${ }^{13}$

Las pruebas posteriores de las investigaciones de Lunney y Ferguson demostraron que el nivel de comodidad de los residentes había aumentado gracias al tutorial en CD-ROM o en línea. ${ }^{7} \mathrm{La}$ diferencia entre nuestra encuesta y la original fue que no nos fue posible evaluar el nivel de conocimientos sobre SD de los residentes debido a una imposibilidad técnica. Por ejemplo, todas nuestras encuestas son autoadministradas y están sujetas al sesgo de quienes las responden, al igual que los resultados. Además, es posible que los médicos con un interés específico en el SD estuvieran más dispuestos a responder 
que aquellos con un interés menor. La tasa de respuesta fue del $34,0 \%$. Esta podría considerarse una cantidad suficiente de participantes para una investigación en línea a nivel nacional.

Este estudio representa el primer paso para comprender la actitud y la predisposición de los pediatras turcos para recibir formación sobre cómo dar el diagnóstico de SD a las familias. La comunicación es una habilidad que se aprende, no un rasgo de la personalidad. ${ }^{14-16}$ Para brindar una buena vida a las familias y permitirles la adaptación al diagnóstico, ante todo, los pediatras deben estar muy bien informados acerca del SD y las intervenciones relacionadas. ${ }^{5}$ Existe la necesidad sustancial de recibir capacitación formal para mejorar los niveles de comodidad de los pediatras al dar el diagnóstico de SD. Los programas de residencia pediátrica deben incorporar capacitación para dar malas noticias a las familias de los bebés con SD, más allá de los conocimientos brindados a los estudiantes en pos del desarrollo de competencias y habilidades. ${ }^{5}$

Investigaciones adicionales sobre qué tipo de intervenciones educativas tienen una mayor influencia en el cambio de actitud de los médicos al dar malas noticias ayudarían a promocionar su adopción diseminada en la formación médica. Se ha informado que los tutoriales en línea, ${ }^{8}$ el desarrollo de habilidades basadas en simulaciones ${ }^{17}$ o competencias, ${ }^{18}$ y los planes de estudio con talleres son útiles y se ha demostrado su eficacia en estudios realizados en residentes de pediatría.

Sentirse más cómodo durante la comunicación será útil, no solo en el contexto del SD sino también para dar otros diagnósticos, como espina bífida, autismo y síndrome del cromosoma $X$ frágil. $^{8}$

\section{CONCLUSIÓN}

Este estudio mostró que los residentes de pediatría tuvieron un nivel de incomodidad alto cuando comunicaban el diagnóstico de SD a los padres del recién nacido. Las residentes tuvieron un nivel de incomodidad significativamente mayor que los residentes.

Existe la necesidad de recibir capacitación formal durante la formación en la facultad de medicina para mejorar el nivel de comodidad de los pediatras turcos al dar un diagnóstico de SD y de aplicar dicha capacitación durante la formación médica continua.

\section{REFERENCIAS}

1. Parker SE, Mai CT, Canfield MA, Rickard R, et al. Updated national birth prevalence estimates for selected birth defects in the United States, 2004-2006. Birth Defects Res A Clin Mol Teratol 2010;88(12):1008-16.

2. Skotko BG. Prenatally diagnosed Down syndrome: mothers who continued their pregnancies evaluate their health care providers. Am J Obstet Gynecol 2005;192(3):670-7.

3. Carr J. Six weeks to twenty-one years old: a longitudinal study of children with Down's syndrome and their families. Third Jack Tizard memorial lecture. JChild Psychol Psychiatry 1988;29(4):407-31.

4. Skotko B. Mothers of children with Down syndrome reflect on their postnatal support. Pediatrics 2005;115(1):64-77.

5. Skotko BG, Kishnani PS, Capone GT; Down Syndrome Diagnosis Study Group. Prenatal diagnosis of Down syndrome: how best to deliver the news. Am J Med Genet A 2009;149A(11):2361-7.

6. Rosas-Blum E, Shirsat $P$, Leiner M. Communicating genetic information: a difficult challenge for future pediatricians. BMC Med Educ 2007;7:17.

7. Ferguson JE 2nd, Kleinert HL, Lunney CA, Campbell LR. Resident physicians' competencies and attitudes in delivering a postnatal diagnosis of Down syndrome. Obstet Gynecol 2006;108(4):898-905.

8. Lunney CA, Kleinert HL, Ferguson JE 2nd, Campbell L. Effectively training pediatric residents to deliver diagnoses of Down syndrome. Am J Med Genet A 2012;158A(2):384-90.

9. Jefferson L, Bloor K, Birks Y, Hewitt C,et al. Effect of physicians' gender on communication and consultation length: a systematic review and meta-analysis. $J$ Health Serv Res Policy 2013;18(4):242-8.

10. Roter DL, Hall JA, Aoki Y. Physician gender effects in medical communication: a meta-analytic review. JAMA 2002;288(6):756-64.

11. Lynch EC, Staloch NH. Parental perceptions of physicians' communication in the informing process. Ment Retard 1988;26(2):77-81.

12. Hedov G, Wikblad K, Annerén G. First information and support provided to parents of children with Down syndrome in Sweden: clinical goals and parental experiences. Acta Paediatr 2002;91(12):1344-9.

13. Van Riper M, Ryff C, Pridham K. Parental and family well-being in families of children with Down syndrome: a comparative study. Res Nurs Health 1992;15(3):227-35.

14. Simpson M, Buckman R, Stewart M, Maguire P, etal. Doctorpatient communication: the Toronto consensus statement. BMJ 1991;303(6814):1385-7.

15. Kurtz SM. Doctor-patient communication: principles and practices. Can J Neurol Sci 2002;29(Suppl 2):S23-9.

16. Mehta PN.Communication skills--talking to parents. Indian Pediatr 2008;45(4):300-4.

17. Greenberg LW, Ochsenschlager D, O'Donnell R, Mastruserio J, et al. Communicating bad news: a pediatric department's evaluation of a simulated intervention. Pediatrics 1999;103(6 Pt 1):1210-7.

18. Silva DH. A competency-based communication skills workshop series for pediatric residents. Bol Asoc Med P R 2008;100(2):8-12. 